\title{
MICROLOCAL HOLMGREN'S THEOREM FOR A CLASS OF HYPO-ANALYTIC STRUCTURES
}

\author{
S. BERHANU
}

\begin{abstract}
A microlocal version of Holmgren's Theorem is proved for a certain
\end{abstract} class of the hypo-analytic structures of Baouendi, Chang, and Treves.

\section{INTRODUCTION}

In [4] Sjöstrand gave a simpler proof of a result of Schapira [3] concerning a microlocal version of Holmgren's theorem for real analytic data. Inspired by [4], in this paper we will extend Schapira's result to a certain class of hypoanalytic structures. The paper is organized as follows: In $\S 2$ we discuss the Cauchy-Kovalevska theorem for maximal hypo-analytic structures. In $\S 3$ we introduce a class of hypo-analytic structures which we call real hypo-analytic, give a statement of the main theorem of this article, and derive two corollaries. A lemma is included in the same section and is used in the proof of the main theorem which appears in $\S 4$.

\section{Cauchy-Kovalevska for hypo-AnAlytic STRUCTURES}

We are interested in the hypo-analytic structures introduced by Baouendi, Chang, and Treves in [1]. We briefly recall the relevant concepts here.

Let $\Omega$ be a smooth manifold of dimension $m$. A hypo-analytic structure of maximal dimension on $\Omega$ is the data of an open covering $\left\{U_{\alpha}\right\}$ of $\Omega$ and for each index $\alpha$, of $m C^{\infty}$ functions $Z_{\alpha}^{1}, \ldots, Z_{\alpha}^{m}$ satisfying the following two conditions:

(1) $d Z_{\alpha}^{1}, \ldots, d Z_{\alpha}^{m}$ are linearly independent at each point of $U_{\alpha}$;

(2) if $U_{\alpha} \cap U_{\beta} \neq \varnothing$, there are open neighborhoods $O_{\alpha}$ of $Z_{\alpha}\left(U_{\alpha} \cap U_{\beta}\right)$ and $O_{\beta}$ of $Z_{\beta}\left(U_{\alpha} \cap U_{\beta}\right)$ and a holomorphic map $F_{\beta}^{\alpha}$ of $O_{\alpha}$ onto $O_{\beta}$ such that $Z_{\beta}=F_{\beta}^{\alpha} \circ Z_{\alpha}$ on $U_{\alpha} \cap U_{\beta}$.

We will use the notation $Z_{\alpha}=\left(Z_{\alpha}^{1}, \ldots, Z_{\alpha}^{m}\right): U_{\alpha} \mapsto C^{m}$. A distribution $h$ defined in an open neighborhood of a point $p_{0}$ of $\Omega$ is hypo-analytic at $p_{0}$ if there is a chart $\left(U_{\kappa}, Z_{\alpha}\right)$ of the above type whose domain contains $p_{0}$ and a

Received by the editors September 11, 1989.

1980 Mathematics Subject Classification (1985 Revision). Primary 35A20; Secondary 35S99.

It is a pleasure to express my gratitude to my teacher and adviser Professor F. Treves. 
holomorphic function $\tilde{h}$ defined on an open neighborhood of $Z_{\alpha}\left(p_{0}\right)$ in $C^{m}$ such that $h=\tilde{h} \circ Z_{\alpha}$ in a neighborhood of $p_{0}$. By a hypo-analytic local chart we mean an $m+1$-tuple $\left(U, Z^{1}, \ldots, Z^{m}\right)$ [abbreviated $(U, Z)$ ] consisting of an open subset $U$ of $\Omega$ and of $m$ hypo-analytic functions whose differentials are linearly independent at every point of $U$.

In [2] we introduced hypo-analytic differential operators which by definition map hypo-analytic functions to hypo-analytic functions. A linear differential operator $P$ on $\Omega$ is hypo-analytic if and only if for every hypo-analytic local chart $\left(U, Z^{1}, \ldots, Z^{m}\right), U$ sufficiently small, and vector fields $M_{1}, \ldots, M_{m}$ satisfying $M_{j} Z^{k}=\delta_{j}^{k}$ we have: $P=\sum_{|\alpha| \leq n} a_{c \alpha}(x) M^{\alpha}$, where each $a_{\alpha k}$ is a hypo-analytic function on $U$. Let $p$ be an arbitrary point of $\Omega$. The differentials of the germs of hypo-analytic functions at $p$ make up a complex vector subspace of the complex cotangent space $C T_{p}^{*} \Omega$. This subspace, which we denote by $T_{p}^{\prime}$, has dimension $=m$. Condition (2) in the definition of hypo-analytic structures implies that the subspace $T_{p}^{\prime}$ makes up a smooth vector subbundle $T^{\prime}$ of the complex cotangent bundle $C T^{*} \Omega . T^{\prime}$ will be referred to as the structure bundle.

We now introduce the concept of hypo-analytic submanifolds. By a submanifold of $\Omega$ we mean a subset of $\Omega$ equipped with a $C^{\infty}$ structure such that the natural injection into $\Omega$ is a $C^{\infty}$ map with injective differential. Let $M$ be a submanifold of $\Omega$. We shall denote by $\pi_{M}$ the natural map $T^{*} \Omega_{\mid M} \mapsto T^{*} M$ and by $\pi_{M}^{C}$ the analogous map of the complex cotangent bundles. In general, $T_{M}^{\prime}=\pi_{M}^{C}\left(T^{\prime}\right)$ is not a vector bundle.

Definition 2.1. A submanifold $M$ of $\Omega$ is called a hypo-analytic submanifold if it is equipped with a hypo-analytic structure whose structure bundle is identical to $T_{M}^{\prime}$ and which has the following property: Given any hypo-analytic function $f$ on an open set $\Omega^{\prime} \subset \Omega$ which intersects $M$, the restriction of $f$ to $M \cap \Omega^{\prime}$ is hypo-analytic.

Simple examples show that the second property in the above definition is not redundant.

Proposition 2.1. Suppose $\Sigma$ is a hypo-analytic submanifold of $\Omega$ whose structure bundle has dimension $m-k$. Then each point $q \in \Sigma$ is contained in a hypoanalytic chart $\left(U ; Z^{1}, \ldots, Z^{m}\right)$ of $\Omega$ with $Z^{m-k+1}, \ldots, Z^{m}$ all vanishing on $U \cap \Sigma$.

Proof. Let $q \in \Sigma$ and $\left(U ; W^{1}, \ldots, W^{m}\right)$ be a hypo-analytic chart for $\Omega$ around $q$. Since the differentials $d W^{1}, \ldots, d W^{m}$ span $C T^{*} U$, without loss of generality we may assume that $\pi_{\Sigma}^{C}\left(d W^{1}\right), \ldots, \pi_{\Sigma}^{C}\left(d W^{m-k}\right)$ span $C T^{*}(U \cap \Sigma)$.

Moreover, $\left(U \cap \Sigma, W_{\mid \Sigma}^{1}, \ldots, W_{\mid \Sigma}^{m-k}\right)$ is a hypo-analytic chart in $\Sigma$ since $\Sigma$ is a hypo-analytic submanifold of $\Omega$. 
Now $W^{m-k+1}, \ldots, W^{m}$ all restrict to hypo-analytic functions in $\Sigma$. Therefore, there are holomorphic functions $H_{1}, \ldots, H_{k}$ such that $W^{m-k+j}(x)=$ $H_{j}\left(W^{1}(x), \ldots, W^{m-k}(x)\right)$ for each $x \in \Sigma \cap U$ and $1 \leq j \leq k$. Here the set $U$ may have to be contracted. For $x \in U$, let

$$
Z^{j}(x)=W^{j}(x), \quad 1 \leq j \leq m-k,
$$

and

$$
Z^{l}(x)=W^{m-k+l}(x)-H_{l}\left(W^{1}(x), \ldots, W^{m-k}(x)\right)
$$

when $m-k \leq l \leq m$.

Then $\left(U ; Z^{1}, \ldots, Z^{m}\right)$ is a hypo-analytic chart on $\Omega$ satisfying the properties in the proposition.

Remark 2.1. If $\Sigma$ is a hypo-analytic submanifold of $\Omega$, then the dimension of $\Sigma$ is the same as the dimension of its structure bundle.

Suppose now $P$ is a hypo-analytic differential operator on $\Omega$. We would like to introduce the concept of noncharacteristic hypersurfaces. Let $\Sigma$ be a hypo-analytic hypersurface of $\Omega$. By Proposition $2.1, \Sigma$ is locally given by $H(x)=0$, where $H$ is hypo-analytic and $d H \neq 0$. If $\left(U ; Z^{1}, \ldots, Z^{m}\right)$ is a hypo-analytic chart for $\Omega$ near a central point $q \in \Sigma$, then $P$ can be written as $P=\sum_{|\alpha| \leq k} a_{o x}(Z(x)) M^{\alpha}$ and $H(x)=\tilde{H}(Z(x))$ for some holomorphic functions $a_{x x}$ and $\tilde{H}$ in a neighborhood of $Z(q)$ in $C^{m}$. We push everything by the map $Z$ into $C^{m}$ near $Z(q)$ and write $P^{Z}\left(z, \frac{\partial}{\partial z}\right)=\sum_{|\alpha| \leq k} a_{\alpha}(z)\left(\frac{\partial}{\partial z}\right)^{\alpha}$ and $\Sigma^{Z}=\left\{z \in C^{m}: \tilde{H}(z)=0\right\}$.

Since $d H \neq 0, \Sigma^{Z}$ is a complex submanifold of $C^{m}$ of complex codimension 1 passing through $Z(q)$.

If $\left(V ; W^{1}, \ldots, W^{m}\right)$ is another hypo-analytic chart about $q$, let $G$ be a biholomorphism near $Z(q)$ in $C^{m}$ such that $\left(W^{1}, \ldots, W^{m}\right)=G\left(Z^{1}, \ldots, Z^{m}\right)$. Then $P_{k}^{W}\left(w, \frac{\partial}{\partial w}\right)$ and $\Sigma^{W}$ are the expressions of $P_{k}^{Z}\left(z, \frac{\partial}{\partial z}\right)$ and $\Sigma^{Z}$ in the coordinates $w^{1}, \ldots, w^{m}$ of $C^{m}$. Hence, in particular, $\Sigma^{Z}$ is noncharacteristic with respect to $P^{Z}$ if and only if $\Sigma^{W}$ is noncharacteristic with respect to $P^{W}$.

This observation justifies the following definition in which we use the same notations as above.

Definition 2.2. We say $\Sigma$ is noncharacteristic with respect to $P$ at a point $q \in \Sigma$ if $\Sigma^{Z}$ is noncharacteristic with respect to $P^{Z}\left(z, \frac{\partial}{\partial z}\right)$ at $Z(q)$ for some hypo-analytic chart $\left(U ; Z^{1}, \ldots, Z^{m}\right)$ about $q$.

We can now formulate a Cauchy-Kovalevska theorem for a hypo-analytic differential operator and hypo-analytic Cauchy data on a noncharacteristic hypoanalytic hypersurface.

Suppose now $P$ is a hypo-analytic differential operator and $\Sigma$ is a noncharacteristic hypo-analytic hypersurface with respect to $P$ at the point $q \in \Sigma$. Let 
the order of $P$ near $q=k$. Suppose $L$ is a hypo-analytic vector field not belonging to $C T \Sigma$ at the point $q$ (and hence near $q$ ). Then we have:

Theorem 2.1. There is an open neighborhood $\Omega^{\prime}$ of $q$ in $\Omega$ such that to every hypo-analytic function $f$ in $\Omega^{\prime}$ and to every set of $k$ hypo-analytic functions $u_{0}, \ldots, u_{k-1}$ on $\Sigma \cap \Omega^{\prime}$, there is a unique hypo-analytic function $u$ in $\Omega^{\prime}$ such that

$$
P u=f \quad \text { in } \Omega^{\prime},
$$

and for every $j=0, \ldots, k-1, L^{j} u=u_{j}$ in $\Sigma \cap \Omega^{\prime}$.

Proof. By Proposition 2.1, $q \in \Sigma$ is contained in a hypo-analytic chart $\left(U ; Z^{1}\right.$, $\ldots, Z^{m}$ ) of $\Omega$ with $Z^{m}$ vanishing on $U \cap \Sigma$. Let $M_{1}, \ldots, M_{m}$ be the vector fields in $U$ satisfying $M_{j} Z^{k}=\delta_{j}^{k}$. Then in the chart $(U, Z)$, we may write $P=\sum_{|\alpha| \leq k} a_{\alpha}(x) M^{(k}$ and $L=\sum_{j} c_{j}(x) M_{j}$, where the coefficients are all hypo-analytic. The condition $L \notin C T \Sigma$ near $q$ is equivalent to $c_{m}(x) \neq 0$ for $x$ near $q$.

Let $\tilde{u}_{j}, \tilde{f}, \tilde{a}_{\alpha}$, and $\tilde{c}_{j}$ be the holomorphic functions defined near $Z(q) \in$ $C^{m}$ such that $u_{j}(x)=\tilde{u}_{j}(Z(x))$ etc.

Set

$$
\begin{gathered}
P^{Z}\left(z, \frac{\partial}{\partial z}\right)=\sum_{|\alpha| \leq k} a_{\alpha}(z)\left(\frac{\partial}{\partial z}\right)^{\alpha}, \\
L^{Z}=\sum_{j=1}^{m} \tilde{c}_{j}(z) \frac{\partial}{\partial z_{j}} \text { and } \Sigma^{Z}=\left\{z \in C^{m}: z_{m}=0\right\} .
\end{gathered}
$$

The assumptions on $\Sigma$ and $L$ imply that $\Sigma^{Z}$ is noncharacteristic for $P^{Z}$ and that $\tilde{c}_{m}(z) \neq 0$ for $z$ near $Z(q)$. Therefore the existence part of Theorem 2.1 follows from the existence part of the holomorphic version of the CauchyKovalevska theorem applied to the problem

$$
P^{Z} \tilde{u}=\tilde{f} \quad \text { near } Z(q) \text { in } C^{m}
$$

and for $0 \leq j \leq k-1$,

$$
\left(L^{Z}\right)^{j} \tilde{u}=\tilde{u}_{j} \quad \text { near } Z(q) \text { in } \Sigma^{Z}(\text { see [7]). }
$$

We just set $u(x)=\tilde{u}(Z(x))$ and observe that $M_{j} u(x)=\frac{\partial \tilde{u}}{\partial z_{j}}(Z(x))$ for each $j=1, \ldots, m$. To see the uniqueness, suppose $u^{\prime}$ is another solution and set $v=u-u^{\prime}$. Then

$$
P v=0 \text { in } \Omega^{\prime} \quad \text { and } \quad L^{j} v=0 \text { in } \Sigma \cap \Omega^{\prime}
$$

and $v$ is hypo-analytic. Since $M_{1}, \ldots, M_{m-1}$ all belong to $C T \Sigma$ and $v=0$ on $\Sigma$, it follows that $M_{1} v=\cdots=M_{m-1} v=0$ on $\Sigma$ (near $q$ ). Now $L=$ $\sum_{j=1}^{m} c_{j}(x) M_{j}$ with $c_{m}(x) \neq 0$ and $L v=0$ on $\Sigma$. Therefore $M_{m} v=0$ on $\Sigma$. Moreover, from $L^{j} v=0$ for $0 \leq j \leq k-1$, we deduce that $M^{\text {(x }} v=0$ for $|\alpha| \leq k-1$ on $\Sigma$. Next, since the coefficient of $M_{m}^{k}$ in $P=\sum_{|\alpha| \leq k} a_{r \gamma}(x) M^{(k}$ 
is nonzero, it follows that on $\Sigma, M^{\alpha} v=0$ for $|\alpha| \leq k$. Finally, applying the vector fields $M_{j}$ to the equation $P v=0$, we see that $M^{\alpha} v=0$ on $\Sigma$ for all indices $\alpha$. Now let $\tilde{v}$ be the holomorphic function near $Z(q)$ in $C^{m}$ satisfying $v(x)=\tilde{v}(Z(x))$.

We write the power series of $v$ around $Z(q)$ as

$$
\tilde{v}(z)=\sum a_{\alpha}(z-Z(q))^{\alpha}, \quad \text { where } a_{\alpha}=\frac{1}{\alpha !}\left(\frac{\partial}{\partial z}\right)^{\alpha} \tilde{v}(Z(q)) .
$$

But then

$$
\left(\frac{\partial}{\partial z}\right)^{\alpha} \tilde{v}(Z(q))=\left(M^{\alpha} v\right)(q)=0 \quad \forall \alpha .
$$

Therefore, $\tilde{v} \equiv 0$ near $Z(q)$. Hence $v \equiv 0$ in $\Omega^{\prime}$.

\section{REAL HYPO-ANALYTIC STRUCTURES AND STATEMENT OF THE MAIN RESULT}

We will continue to look at a maximal hypo-analytic structure on $\Omega$. We noted that a hypersurface $\Sigma$ is hypo-analytic if and only if $\Sigma$ is the zero set of a hypo-analytic function $f$ with nonzero differential. We now strengthen this condition and introduce the following:

Definition 3.1. $\Sigma$ is said to be a real hypo-analytic hypersurface if every point $p \in \Sigma$ has a neighborhood $U_{p}$ in $\Omega$, a hypo-analytic function $h$ of a nonzero differential defined on $U_{p}$, and $\varepsilon>0$ such that:

(1) $\Sigma \cap U_{p}=\left\{x \in U_{p}: h(x)=0\right\}$.

(2) For $c \in C,|c|<\varepsilon$, the set $\Sigma_{c}=\left\{x \in U_{p}: h(x)=c\right\}$ is either $\varnothing$ or a hypersurface.

(3) $U \Sigma_{c}$ is a neighborhood in $U_{p}$ of $p ;|c|<\varepsilon$.

We note that near each point of $\Sigma$, the above definition gives a local foliation of $\Omega$ by means of hypo-analytic hypersurfaces.

Example 1. Suppose $\Omega$ is a real analytic structure. The real analytic structure can be viewed as a hypo-analytic structure and in this case, any real analytic hypersurface is real hypo-analytic.

Example 2. Consider a hypo-analytic local chart $(U, Z)$ around 0 in a maximal hypo-analytic structure on $R^{m}$. Suppose $Z_{j}=x_{j}+\sqrt{-1} \phi_{j}(x), j=$ $1, \ldots, m-1$, and $Z_{m}=x_{m}+\sqrt{-1} \phi_{m}\left(x_{m}\right)$, where $\phi=\left(\phi_{1}, \ldots, \phi_{m}\right)$ is real-valued, with zero differential at 0 , and $\phi(0)=0$.

Assume that $U$ is small enough so that the mapping $Z=\left(Z_{1}, \ldots, Z_{m}\right)$ : $U \rightarrow C^{m}$ is a diffeomorphism of $U$ onto $Z(U)$. Then $\Sigma=\left\{x \in U: x_{m}=0\right\}$ is a real hypo-analytic hypersurface. In this case, the defining function can be taken to be $Z_{m}$.

Lemma 3.2 will show that Example 2 is a typical example. 
The proof of the main theorem will use two equivalent formulations of microlocal hypo-analyticity that were developed in [1]. We briefly recall them here.

Sato's Microlocalization. We consider a hypo-analytic local chart $(U, Z)$ of the maximal structure $\Omega$.

In the sequel $\Gamma$ is a nonempty, acute, and open cone in $R^{m} \backslash\{0\}$. For $A$ an open subset of $U$ and $\delta>0$, let

$$
N_{\delta}(A, \Gamma)=\left\{Z(x)+\sqrt{-1} Z_{x}(x) v: x \in A, v \in \Gamma,|v|<\delta\right\} .
$$

Let $B_{\delta}(A, \Gamma)$ denote the space of holomorphic functions on $N_{\delta}(A, \Gamma)$ of tempered growth. More precisely, a holomorphic function $f$ with domain $N_{\delta}(A, \Gamma)$ is in $B_{\delta}(A, \Gamma)$ if it satisfies the condition: to every compact subset $K$ of $N_{\delta}(A, \Gamma)$ there are an integer $k \geq 0$ and a constant $c>0$ such that $|f(z)| \leq c(\operatorname{dist}[z, Z(A)])^{-k}$ for all $z$ in $K$.

In [1] it was shown that if $A$ is sufficiently small and $f \in B_{\delta}(A, \Gamma)$, then for every $\psi \in C_{c}^{\infty}(A)$,

$$
\lim _{t \rightarrow+0} \int_{A} f\left(Z(x)+\sqrt{-1} Z_{x}(x) t v\right) \psi(x) d Z(x)
$$

exists and is independent of $v \in \Gamma$. Let $b f$ denote the limit distribution.

Definition 3.2. Let $u \in D^{\prime}(U)$ and $(x, \xi) \in U \times R_{m} \backslash\{0\}$. We say that $u$ is microlocally hypo-analytic at $(x, \xi)$ if there are an open neighborhood $A \subseteq U$ of $x, \delta>0$ and a finite collection of nonempty acute open cones $\Gamma_{k}$ in $R_{m} \backslash\{0\} \quad(k=1, \ldots, r)$ satisfying $\langle v, \xi\rangle<0$ for every $v$ in each $\Gamma_{k}$ and such that the the following hold:

for each $k$ there is $f_{k} \in B_{\delta}\left(A, \Gamma_{k}\right)$ such that in $A$,

$$
u=b f_{1}+\cdots+b f_{r} .
$$

The above definition of microlocal hypo-analyticity in the cotangent space does not depend on the choice of the chart $(U, Z)$ (see [1]).

Definition 3.3. Let $u \in D^{\prime}(\Omega)$. The hypo-analytic wavefront set of the distribution $u$ is denoted by $\mathrm{WF}_{\mathrm{ha}} u$ and is defined as

$$
\mathrm{WF}_{\text {ha }} u=\left\{(x, \xi) \in T^{*} \Omega: u \text { is not hypo-analytic at }(x, \xi)\right\} .
$$

The FBI Transform. We continue to work in a chart $(U, Z)$ of the maximal structure $\Omega$. Assume that $Z=\left(Z_{1}, \ldots, Z_{m}\right): U \rightarrow C^{m}$ is a diffeomorphism of $U$ onto $Z(U)$ and that $U$ is the domain of local coordinates $x_{j} \quad(1 \leq$ $j \leq m$ ) all vanishing at a "central point" which will be denoted by 0 . We will suppose $Z(0)=0$ and by substituting $Z_{x}(0)^{-1} Z(x)$ for $Z(x)$ if necessary, we may assume that

$$
Z_{x}(0)=\text { the identity matrix. }
$$


Let $u$ be a compactly supported distribution in $U$. We shall refer to

$$
F(u, z, \zeta)=\int_{y} \exp \left(\sqrt{-1} \zeta \cdot(z-Z(y))-\langle\zeta\rangle(z-Z(y))^{2}\right) u(y) d Z(y)
$$

as the Fourier-Bros-Iagolnitzer (in short, FBI) transform of $u$. Here $z \in$ $C^{m}, \zeta \in C_{m}$ with $|\operatorname{Im} \zeta|<|\operatorname{Re} \zeta|$, and

$$
\langle\zeta\rangle^{2}=\zeta_{1}^{2}+\cdots+\zeta_{m}^{2} .
$$

In [1], the authors established the following FBI transform criterion for hypoanalyticity. We will state it here in a form that will be of convenience to us.

Theorem 3.1. The following two properties of a compactly supported distribution are equivalent:

(i) $u$ is microlocally hypo-analytic at $\left(0, \xi^{0}\right) \in T^{*} U \backslash\{0\}$.

(ii) There is an open neighborhood $V$ of 0 in $C^{m}$, a conic open neighborhood $\mathscr{C}_{0}$ of $\xi^{0}$ in $C_{m}$, and constants $c, r>0$ such that $|F(u, z, \zeta)| \leq c \exp (-r|\zeta|)$ for all $z$ in $V$ and for all $\zeta$ in $\mathscr{C}_{0}$.

We are now ready to state the main theorem of this paper.

Theorem 3.2. Let $P$ be a hypo-analytic differential operator and $\Sigma$ a real hypoanalytic hypersurface which is noncharacteristic for $P$. Assume $u \in D^{\prime}(\Omega)$ such that $P u$ is hypo-analytic. Suppose $\left.\sigma \in T^{*} \Omega\right|_{\Sigma}$ for which the hypo-analytic Cauchy data of $u$ are microlocally hypo-analytic at $\pi_{\Sigma}(\sigma)$. Then $\sigma \notin \mathrm{WF}_{\mathrm{ha}} u$.

Remark 3.2. The proof will actually show that it is sufficient to have $P u$ microlocally hypo-analytic at $\sigma$.

From Theorem 3.2 we deduce the following consequences. $\Sigma$ and $P$ will be as in Theorem 3.2.

Corollary 3.1. Suppose $P u$ is hypo-analytic at $q \in \Sigma$ and the hypo-analytic Cauchy data of $u$ is also hypo-analytic at $q$. Then $u$ is hypo-analytic at $q$.

Proof. Since the hypo-analytic Cauchy data is hypo-analytic at $q$, it is microlocally hypo-analytic in every direction in $T_{q}^{*} \Sigma /\{0\}$. (See [1] for a proof.) Therefore, by Theorem 3.2, $u$ is microlocally hypo-analytic in every direction in $T_{q}^{*} \Omega$. Hence by [1], $u$ is hypo-analytic at $q$.

Corollary 3.2. Suppose $P u=0$ and the hypo-analytic Cauchy data of $u$ on $\Sigma$ is 0 . Then $u \equiv 0$.

Proof. By Corollary 3.1, $u$ is hypo-analytic. But then by the uniqueness part of Theorem 2.1,u $\equiv 0$.

The following lemmas will be used in the proof of Theorem 3.2.

Lemma 3.1. Let $P$ be a hypo-analytic differential operator and $\sigma \notin$ Char $P$. If $u \in \mathscr{D}^{\prime}(\Omega)$ for which $\sigma \notin \mathrm{WF}_{\mathrm{ha}} P u$, then $\sigma \notin \mathrm{WF}_{\mathrm{ha}} u$.

Proof. We reason in a chart $(U, Z)$ around 0 where we assume that $Z(0)=$ $0, d Z(0)=\mathrm{Id}, \sigma=\left(0, \xi^{0}\right) \in T^{*} U$, and $U$ is the domain of local coordinates 
$x_{j} \quad(1 \leq j \leq m)$. We can then take $\Re Z_{j}$ as new coordinates in which $Z(x)=$ $x+\sqrt{-1} \phi(x), \phi(0)=0, d \phi(0)=0$ and $\phi=\left(\phi_{1}, \ldots, \phi_{m}\right)$ is real-valued. Moreover, the functions $Z_{j}$ may be selected so that all the derivatives of order 2 of the $\phi_{j}$ vanish at 0 . Indeed, if this is not already so it suffices to replace each $Z_{j}$ by

$$
Z_{j}-\frac{\sqrt{-1}}{2} \sum_{k=1}^{m} \sum_{l=1}^{m} \frac{\partial^{2} \phi_{j}}{\partial x_{k} \partial x_{l}}(0) Z_{k} Z_{l} .
$$

Let $M_{j} \quad(1 \leq j \leq m)$ be the vector fields satisfying $M_{j} Z_{k}=\delta_{j}^{k}$. To prove the lemma, we will use the FBI transform. First we note that for any $f \in C^{1}(U)$,

$$
\left\langle d f, M_{k}\right\rangle=M_{k} f=\sum_{j}\left\langle\left(M_{j} f\right) d Z_{j}, M_{k}\right\rangle \quad \forall k .
$$

It follows that

$$
d f=\sum_{j=1}^{m}\left(M_{j} f\right) d Z_{j} .
$$

Therefore, if $g$ or $h$ has compact support in $U$, by Stokes' theorem we have

$$
\begin{aligned}
0 & =\int_{\partial U} h g d Z_{1} \wedge \cdots \wedge \widehat{d Z}_{j} \wedge \cdots \wedge d Z_{m} \\
& =(-1)^{j-1}\left[\int_{U}\left[\left(M_{j} h\right) g+h\left(M_{j} g\right)\right] d Z_{1} \wedge \cdots \wedge d Z_{m}\right]
\end{aligned}
$$

Hence

$$
\int_{U}\left(M_{j} h\right) g d Z_{1} \wedge \cdots \wedge d Z_{m}=-\int_{U} h\left(M_{j} g\right) d Z_{1} \wedge \cdots \wedge d Z_{m}
$$

If $U$ is sufficiently small, in the chart $(U, Z)$ we may write

$$
P=\sum_{|\alpha| \leq k} a_{\alpha}(x) M^{(k},
$$

where each $a_{\alpha}$ is hypo-analytic on $U$.

Since $\sigma=\left(0, \xi^{0}\right) \notin \mathrm{WF}_{\mathrm{ha}} P u$, Theorem 3.1 tells us that

$$
\begin{aligned}
F & (P u, z, \zeta) \\
& =\int_{U} \exp \left(\sqrt{-1} \zeta \cdot(z-Z(y))-\langle\zeta\rangle(z-Z(y))^{2}\right) \sum_{|\alpha| \leq k} a_{\alpha}(y) M^{(k} u(y) d Z(y)
\end{aligned}
$$

has an exponential decay for $z$ near 0 and $\zeta$ in a complex conic neighborhood of $\xi^{0}$.

Since $y \mapsto \exp \left(\sqrt{-1} \zeta \cdot(z-Z(y))-\langle\zeta\rangle(z-Z(y))^{2}\right)$ is hypo-analytic, for each $j=1, \ldots, m$,

$$
M_{j}(\exp h(z, \zeta, y))=\left[-\sqrt{-1} \zeta_{j}+2\langle\zeta\rangle\left(z_{j}-Z_{j}(y)\right)\right] \exp (h(z, \zeta, y)),
$$


where

$$
h(z, \zeta, y)=\sqrt{-1} \zeta \cdot(z-Z(y))-\langle\zeta\rangle(z-Z(y))^{2}
$$

This observation together with the integration by parts formula (3.1) imply the existence of a hypo-analytic amplitude $Q(z, \zeta, y)$ elliptic at $\sigma$ such that

$$
\begin{aligned}
F(P u, z, \zeta) & \\
& =\int_{U} \exp \left(\sqrt{-1} \zeta \cdot(z-Z(y))-\langle\zeta\rangle(z-Z(y))^{2}\right) Q(z, \zeta, y) u(y) d Z .
\end{aligned}
$$

By the results of [5], we conclude that $\sigma \notin \mathrm{WF}_{\text {ha }} u$.

Lemma 3.2. Suppose $\Sigma$ is a real hypo-analytic hypersurface of $\Omega$. Then each point $p \in \Sigma$ is contained in a hypo-analytic chart $\left(U, Z_{1}, \ldots, Z_{m}\right)$, where $U$ is the domain of local coordinates $\left(U, x_{1}, \ldots, x_{m}\right)$ in which

$$
Z_{j}=x_{j}+\sqrt{-1} \phi_{j}(x) \quad \text { for } 1 \leq j<m
$$

and $Z_{m}=x_{m}+\sqrt{-1} \Psi\left(x_{m}\right)$, where

$\left(\phi_{1}, \ldots, \phi_{m-1}, \Psi\right)$ is real-valued and $\Sigma \cap U=\left\{x \in U: x_{m}=0\right\}$.

Proof. By Proposition 2.1, there is a chart $(U, Z)$ of $\Omega$ near $p$ such that

$$
\Sigma \cap U=\left\{x: Z_{m}(x)=0\right\} \text {. }
$$

Since $d\left(\left.Z_{1}\right|_{\Sigma}\right), \ldots, d\left(\left.Z_{m-1}\right|_{\Sigma}\right)$ are linearly independent, by making linear substitutions if necessary, we may assume that $d\left(\left.\Re Z_{1}\right|_{\Sigma}\right), \ldots, d\left(\left.\Re Z_{m-1}\right|_{\Sigma}\right)$ are independent.

We may then take $\Re Z_{1}, \ldots, \Re Z_{m-1}$ as coordinates on $\Sigma$. By multiplying $Z_{m}$ by $\sqrt{-1}$ if necessary, we may also assume that $\Re Z_{1}, \ldots, \Re Z_{m}$ are coordinates in $U$ (all this locally near $p$ )

Then

$$
Z_{j}=x_{j}+\sqrt{-1} \phi_{j}, \quad Z_{m}=x_{m}+\sqrt{-1} \Psi(x), \quad 1 \leq j<m,
$$

and since $\left.Z_{m}\right|_{\Sigma \cap U^{\prime}}=0$, we have

$$
\Sigma \cap U=\left\{x \in U: x_{m}=0\right\} .
$$

Next let $h$ be the defining function of $\Sigma$ near $p$ satisfying the conditions of Definition 3.1. Write $h(x)=\tilde{h}(Z(x))$, where $\tilde{h}$ is holomorphic.

Since $\left.h\right|_{\Sigma}=\left.\tilde{h}\left(Z_{1}, \ldots, Z_{m-1}, 0\right)\right|_{\Sigma}=0$ and the image of $\Sigma$ under $\left(Z_{1}, \ldots\right.$, $Z_{m-1}$ ) is a totally real manifold of maximal dimension in $C^{m-1}$, it follows that

$$
h(x)=\tilde{h}\left(Z_{1}(x), \ldots, Z_{m}(x)\right)=\tilde{h}\left(Z_{m}(x)\right) .
$$

Now since $d h \neq 0, \tilde{h}$ is invertible. Hence, for any constant $c \in C$,

$$
h(x)=c \quad \text { iff } Z_{m}(x)=\tilde{h}^{-1}(c) .
$$

It now follows from Definition 3.1 that $Z_{m}=x_{m}+\sqrt{-1} \Psi\left(x_{m}\right)$. 


\section{Proof of Theorem 3.2}

Lemma 3.2 permits us to reason in a local hypo-analytic chart $(U, Z)$, where $U$ is also the domain of local coordinates $\left(U, x_{1}, \ldots, x_{m}\right)$ centered at 0 with $Z_{j}=x_{j}+\sqrt{-1} \phi_{j}(x), 1 \leq j<m, Z_{m}=x_{m}+\sqrt{-1} \Psi\left(x_{m}\right), \Sigma$ is given by $x_{m}=0$ and $\sigma=\left(0, \xi_{0}\right)$.

We may also assume that $Z(0)=0, d Z(0)=\mathrm{Id}, \phi^{\prime \prime}(0)=0$, and $\Psi^{\prime \prime}(0)=0$.

Let $M_{j} \quad(1 \leq j \leq m)$ be the vector fields satisfying $M_{j} Z_{k}=\delta_{j}^{k}$. If $p \in \Sigma$ and $1 \leq j<m$, then $\left(M_{j}\right)_{p} \in C T_{p} \Sigma$. Moreover, after multiplication by a nonvanishing hypo-analytic function, $P$ will have the form

$$
P=M_{m}^{n}+\sum_{|\alpha| \leq n, \alpha_{m}<n} a_{\alpha}(x) M^{\alpha},
$$

where the $a_{\alpha}$ are all hypo-analytic functions. Since $P u$ is hypo-analytic, it follows that $u$ is a $C^{\infty}$ function of $x_{m}$ valued in the space of distributions in the variable $x^{\prime}=\left(x_{1}, \ldots, x_{m-1}\right)$ (see [8]). In particular, the trace of $u$ on $\Sigma$ is well defined.

We may therefore restate the theorem as:

Suppose $P u$ is hypo-analytic and $\left(0^{\prime}, \xi^{\prime}\right) \in T_{0^{\prime}}^{*} \Sigma$ such that $\left(0^{\prime}, \xi_{0}^{\prime}\right) \notin$ $\mathrm{WF}_{\mathrm{ha}}\left(M_{m}^{j} u\left(x^{\prime}, 0\right)\right)$ for $0 \leq j<n$. Then $\left(0,\left(\xi_{0}^{\prime}, \xi_{n}\right)\right) \notin \mathrm{WF}_{\mathrm{ha}} u$.

Since the statement is purely local, we may assume that the support of $u$ is contained in a set of the form

$$
\left\{x^{\prime}:\left|x^{\prime}\right| \leq T / 2\right\} \times(-T, T) \text { and }\left\{\left(x^{\prime}, 0\right):\left|x^{\prime}\right| \leq T\right\} \subseteq \Sigma .
$$

For $t \in(-T, T)$, let $\Sigma_{t}=\Sigma \times\{t\}$ and $\Omega_{t}=\left\{\left(x^{\prime}, x_{m}\right):\left|x^{\prime}\right|<T / 2,0 \leq\right.$ $x_{m}<t$ or $\left.t<x_{m} \leq 0\right\}$.

We observe that for any $j, k$, and $l$,

$$
M_{j}\left(M_{k} Z_{l}\right)=0=M_{k}\left(M_{j} Z_{l}\right) .
$$

Since the differentials $d Z_{1}, \ldots, d Z_{m}$ span $C T^{*} U$, it follows that the vector fields $M_{j}$ commute pairwise. This observation together with the integration by parts formula of $\S 3$ and the fact that for each $t$ and $j<m, M_{j} \in C T \Sigma_{t}$ yield:

$$
\begin{aligned}
\left.\int_{\Omega_{t}}(P u) w d Z_{1} \wedge \cdots \wedge d Z_{m}-\int_{\Omega_{t}} u{ }^{t} P w\right) d Z_{1} \wedge \cdots \wedge d Z_{m} \\
=\sum_{j+k \leq n-1} \int_{\Sigma_{l}}\left(M_{m}^{j} u\right)\left(B_{j k}\left(x, M^{\prime}\right) M_{m}^{k} w\right) d Z_{1} \wedge \cdots \wedge d Z_{m-1} \\
\quad-\sum_{j+k \leq n-1} \int_{\Sigma_{0}}\left(M_{m}^{j} u\right)\left(B_{j k}\left(x, M^{\prime}\right) M_{m}^{k} w\right) d Z_{1} \wedge \cdots \wedge d Z_{m-1},
\end{aligned}
$$

where the $B_{j k}$ are hypo-analytic differential operators in $M_{1}, \ldots, M_{m-1}$ of order $n-1-j-k$. 
For $\alpha=\left(z_{0}^{\prime}, \xi^{\prime}\right) \in C^{m-1} \times\left(R_{m-1} \backslash\{0\}\right)$ and $\tau \in C$. satisfying $1<|\tau|<$ $C_{0},|\Im \tau|<\varepsilon \Re \tau$ ( $\varepsilon$ and $C_{0}$ to be determined later), set

$$
V_{\alpha, \tau}\left(z^{\prime}\right)=\exp \left(\sqrt{-1}\left(z_{0}^{\prime}-z^{\prime}\right) \cdot \xi^{\prime}-\tau\left|\xi^{\prime}\right|\left(z_{0}^{\prime}-z^{\prime}\right)^{2}\right) .
$$

Since ${ }^{t} P$ is a hypo-analytic differential operator, let

$$
{ }^{i} \boldsymbol{P}=\sum_{|\alpha| \leq n} c_{\alpha}(x) \boldsymbol{M}^{\alpha},
$$

where each $c_{\alpha}(x)=\tilde{c}_{\alpha}(Z(x))$ for holomorphic $\tilde{c}_{\alpha}$. Set

$$
{ }^{t} P\left(z, \frac{\partial}{\partial z}\right)=\sum_{|\alpha| \leq n} \tilde{c}_{\alpha}(z)\left(\frac{\partial}{\partial z}\right)^{\alpha} .
$$

Let $\tilde{\Sigma}_{t}=\left\{\left(z^{\prime}, t\right) \in C^{m-1} \times\{t\}:\left|z^{\prime}\right| \leq T\right\}$.

The Cauchy-Kovalevska theorem tells us that there is $t_{0}>0$ such that if $t \in\left[-t_{0}, t_{0}\right]$ we can find a solution $\tilde{w}(z)=\tilde{w}_{\alpha, \tau, t}(z)$ in a neighborhood of $\left\{\left(z^{\prime}, x_{m}\right) \in C^{m-1} \times R:\left|z^{\prime}\right| \leq T,\left|x_{m}\right|<t_{0}\right\}$ of the problem

$$
\begin{gathered}
{ }^{t} P\left(z, \frac{\partial}{\partial z}\right) \tilde{w}=0,\left.\quad \tilde{w}\right|_{\tilde{\Sigma}_{t}}=\cdots=\left.\left(\frac{\partial}{\partial z_{m}}\right)^{n-2} \tilde{w}\right|_{\tilde{\Sigma}_{t}}=0 \\
\left.\left(\frac{\partial}{\partial z_{m}}\right)^{n-1} \tilde{w}\right|_{\tilde{\Sigma}_{t}}=V_{\alpha, \tau} .
\end{gathered}
$$

The solution $\tilde{w}=\tilde{w}_{\alpha, \tau, t}$ can be estimated in terms of the Cauchy data on $\tilde{\Sigma}_{t}$. Indeed, the Ovcyannikov method (see [6]) implies

$$
\begin{gathered}
\exists c>0 \text { independent of } t, \tau, \alpha \quad \text { such that } \\
\left|\tilde{w}_{\alpha, \tau, t}\left(w^{\prime}, z_{m}\right)\right| \leq c \sum_{\left|\beta^{\prime}\right| \leq n} \operatorname{Sup}_{z^{\prime}-w^{\prime}|\leq c| z_{m}-t \mid}\left|\partial_{z^{\prime}}^{\beta^{\prime}} V_{\alpha, \tau}\left(z^{\prime}\right)\right| .
\end{gathered}
$$

For $\left|\beta^{\prime}\right| \leq n$ we have

$$
\begin{aligned}
\left|\partial_{z^{\prime}}^{\beta^{\prime}} V_{\alpha, \tau}\left(z^{\prime}\right)\right| \leq c_{1}\left(|1+| \xi^{\prime} \mid\right)^{n} \exp \left(\left\langle\Im\left(z^{\prime}-z_{0}^{\prime}\right), \xi^{\prime}\right\rangle\right. & -\left|\xi^{\prime}\right|\left[\Re \tau\left\{\left(\Re z^{\prime}-\Re z_{0}^{\prime}\right)^{2}\right\}\right. \\
& \left.\left.-2 \Im \tau \Re\left(z^{\prime}-z_{0}^{\prime}\right) \cdot \Im\left(z^{\prime}-z_{0}^{\prime}\right)\right]\right) .
\end{aligned}
$$

We are going to be interested in $z^{\prime}, z_{0}^{\prime}$, where $\Im z^{\prime}$ is small compared to $\Re z^{\prime}$ and $z_{0}^{\prime}$ is close enough to $0^{\prime}$. This consideration together with a sufficiently small choice of $\varepsilon$ in the definition of $\tau$ imply for $\left|\beta^{\prime}\right| \leq n$

$$
\begin{aligned}
& \left|\partial_{z^{\prime}}^{\beta^{\prime}} V_{(r, \tau}\left(z^{\prime}\right)\right| \leq c_{1}\left(|+| \xi^{\prime} \mid\right)^{n} \\
& \quad \cdot \exp \left(\left\langle\Im\left(z^{\prime}-z_{0}^{\prime}\right), \xi^{\prime}\right\rangle-\frac{\Re \tau}{2}\left|\xi^{\prime}\right|\left[\left(\Re z^{\prime}-\Re z_{0}^{\prime}\right)^{2}-\left(\Im z^{\prime}-\Im z_{0}^{\prime}\right)^{2}\right]\right) .
\end{aligned}
$$


Application of (4.5) to (4.3) yields

$$
\begin{aligned}
& \left|\tilde{w}_{\alpha, \tau, t}\left(z^{\prime}, x_{m}+i \Psi\left(x_{m}\right)\right)\right| \\
& \quad \leq c_{1}\left(|+| \xi^{\prime} \mid\right)^{n} \exp \left(\left\langle\Im\left(z^{\prime}-z_{0}^{\prime}\right), \xi^{\prime}\right\rangle-\frac{\Re \tau}{2}\left|\xi^{\prime}\right|\right. \\
& \left.\quad \times\left[\left(\Re z^{\prime}-\Re z_{0}^{\prime}\right)^{2}-\left(\Im z^{\prime}-\Im z_{0}^{\prime}\right)^{2}\right]+c\left|\xi^{\prime}\right|\left|x_{m}-t\right|\right) .
\end{aligned}
$$

Let $w_{\alpha, \tau, t}(x)=\tilde{w}_{\alpha, \tau, t}(Z(x))$. For $\alpha=\left(z_{0}^{\prime}, \xi^{\prime}\right)$ in a sufficiently small conic neighborhood of $\left(0^{\prime}, \xi_{0}^{\prime}\right)$ and with $w=w_{\alpha, \tau, t}$ we wi.. estimate the term

$$
\int_{\Omega_{t}}(P u) w d Z_{1} \wedge \cdots \wedge d Z_{m} \quad \text { in (4.1). }
$$

(4.2) tells us that $w=w_{\alpha, \tau, t}$ solves

$$
{ }^{t} P(x, M) w=0,\left.\quad w\right|_{\Sigma_{l}}=\cdots=\left.M_{m}^{n-2} w\right|_{\Sigma_{l}}=0,
$$

and

$$
M_{m}^{n-1} w\left(x^{\prime}, t\right)=V_{\alpha, \tau}\left(Z\left(x^{\prime}, t\right)\right) .
$$

Since $P u$ and $w=w_{\alpha, \tau, t}$ are hypo-analytic, we can deform the integration contour from $\Omega_{t}$ to the image of $\Omega_{t}$ under the map

$$
\left(x^{\prime}, x_{m}\right) \mapsto \theta\left(x^{\prime}, x_{m}\right)=Z\left(x^{\prime}, x_{m}\right)-\sqrt{-1}\left(d \chi\left(x^{\prime}\right) \frac{\xi^{\prime}}{\left|\xi^{\prime}\right|}, 0\right),
$$

where $\chi\left(x^{\prime}\right)$ is a cutoff function $\equiv 1$ near $\Re z_{0}^{\prime}$ and $d$ is chosen so that we stay inside the domain of hypo-analyticity.

Along this contour, (4.6) gives the following estimate on $w=w_{\alpha, \tau, t}$ :

$$
\begin{aligned}
|w| & \leq c_{1}\left(|+| \xi^{\prime} \mid\right)^{n} \\
& \times e^{\left(-d x\left(x^{\prime}\right)\left|\xi^{\prime}\right|+\left\langle\phi^{\prime}(x), \xi^{\prime}\right\rangle-\frac{\Re r}{2}\left|\xi^{\prime}\right|\left[\left(x^{\prime}-\Re z_{0}^{\prime}\right)^{2}\left(\phi^{\prime}(x)-d x\left(x^{\prime}\right) \frac{\xi^{\prime}}{\left|\xi^{\prime}\right|}-\Im z_{0}^{\prime}\right)^{2}\right]+c\left|\xi^{\prime}\right|\left|x_{m}-t\right|\right) .}
\end{aligned}
$$

$\left(\right.$ Here $\phi^{\prime}=\left(\phi_{1}, \ldots, \phi_{m-1}\right)$.)

By using the term $\left(x^{\prime}-\Re z_{0}^{\prime}\right)^{2}$ when $x^{\prime}$ is away from $\Re z_{0}^{\prime}$ and the term $d \chi\left(x^{\prime}\right)\left|\xi^{\prime}\right|$ when $x^{\prime}$ is near $\Re z_{0}^{\prime}$, we see that $w$ is exponentially decaying along this contour. The latter may require shrinking of the interval $\left[-t_{0}, t_{0}\right]$ to a smaller interval which we will still call $\left[-t_{0}, t_{0}\right]$.

It follows that we can find a sufficiently small $t>0$ and a sufficiently large $c_{2}>0$ such that

$$
\left|\int_{\Omega_{t}}(P u) w_{\alpha, \tau, t} d Z_{1} \wedge \cdots \wedge d Z_{m}\right| \leq c_{2} \exp \left(-\frac{\left|\xi^{\prime}\right|}{c_{2}}\right)
$$

for $|t| \leq t_{0}$ and $\alpha=\left(z_{0}^{\prime}, \xi^{\prime}\right)$ in a small conic neighborhood of $\left(0^{\prime}, \xi_{0}^{\prime}\right)$. 
Since $w=w_{\alpha, \tau, t}$ solves $\left(4.2^{\prime}\right)$, formula (4.1) reduces to

$$
\begin{aligned}
& i(-1)^{n+1} \int_{\Omega_{1}}(P u) w d Z_{1} \wedge \cdots \wedge d Z_{m} \\
& =\int_{\left|x^{\prime}\right| \leq T} e^{\left(\sqrt{-1}\left\langle z_{0}^{\prime}-Z^{\prime}\left(x^{\prime}, t\right), \xi^{\prime}\right\rangle-\tau\left|\xi^{\prime}\right|\left(z_{0}^{\prime}-Z^{\prime}\left(x^{\prime}, t\right)\right)^{2}\right)} \\
& \quad \times u\left(x^{\prime}, t\right) d Z_{1} \wedge \cdots \wedge d Z_{m-1}\left(x^{\prime}, t\right) \\
& \quad+i(-1)^{n} \sum_{j+k \leq n-1} \int_{\Sigma_{0}}\left(M_{m}^{j} u\right)\left(B_{j k}\left(x, M^{\prime}\right) M_{m}^{k} w\right) d Z_{1} \wedge \cdots \wedge d Z_{m-1}\left(x^{\prime}, 0\right) .
\end{aligned}
$$

We consider now the integrals over $\Sigma_{0}=\Sigma$. Fix $j$ and $k \ni j+k \leq n-1$. Since by assumption $\left(0^{\prime}, \xi^{\prime}\right) \notin \mathrm{WF}_{\mathrm{ha}}\left(\left.M_{m}^{j} u\right|_{\Sigma_{0}}\right)$, without loss of generality we may assume

$$
\left.M_{m}^{j} u\right|_{\Sigma_{0}}=\lim _{s \downarrow 0} f_{j}\left(Z^{\prime}\left(x^{\prime}, 0\right)+\sqrt{-1} s Z_{x^{\prime}}^{\prime}\left(x^{\prime}, 0\right) v\right)
$$

$\left(Z^{\prime}=\left(Z_{1}, \ldots, Z_{m-1}\right)\right)$ for some tempered holomorphic function $f_{j}$, and $v$ is in a cone $\Gamma_{j} \subseteq R^{m-1}$ satisfying

$$
\left\langle v, \xi_{0}^{\prime}\right\rangle<0
$$

Hence, in the integral over $\Sigma_{0}$, we may deform a contour to $Z\left(x^{\prime}, 0\right)+$ $\sqrt{-1} s \chi\left(x^{\prime}\right) Z_{x}\left(x^{\prime}, 0\right) v$, where $s$ is chosen sufficiently small and $\chi\left(x^{\prime}\right)$ is selected as before.

Estimates analogous to (4.6) are also valid for the derivatives $\left\{M_{m}^{k} w\right\}_{k}$. Such estimates and the new contour for each $j$ yields, after enlarging $c_{2}$ if necessary,

$$
\left|\int_{\Sigma_{0}}\left(M_{m}^{j} u\right) B_{j k}\left(x, M^{\prime}\right) M_{m}^{k} w d Z_{1} \wedge \cdots \wedge d Z_{m-1}\right| \leq c_{2} \exp \left(-\frac{\left|\xi^{\prime}\right|}{c_{2}}\right)
$$

for $t \in\left[-t_{0}, t_{0}\right]$ and $\alpha=\left(z_{0}^{\prime}, \xi_{0}^{\prime}\right)$ in a small conic neighborhood of $\left(0^{\prime}, \xi_{0}^{\prime}\right)$.

It follows that (after modifying $t_{0}$ and $c_{2}$ )

$$
\begin{gathered}
\left|\int_{\left|x^{\prime}\right| \leq T} u\left(x^{\prime}, t\right) \exp \left(\sqrt{-1}\left\langle z_{0}^{\prime}-Z^{\prime}\left(x^{\prime}, t\right), \xi^{\prime}\right\rangle-\tau\left|\xi^{\prime}\right|\left(Z^{\prime}\left(x^{\prime}, t\right)-z_{0}^{\prime}\right)^{2}\right) d Z^{\prime}\right| \\
\leq c_{2} \exp \left(-\frac{\left|\xi^{\prime}\right|}{c_{2}}\right)
\end{gathered}
$$

for $t \in\left[-t_{0}, t_{0}\right]$ and $\alpha=\left(z_{0}^{\prime}, \xi^{\prime}\right)$ in a small conic neighborhood of $\left(0^{\prime}, \xi_{0}^{\prime}\right)$.

Let $I\left(t, \tau, z_{0}^{\prime}, \xi^{\prime}\right)=$ the integral (without the absolute value) in (4.11). Suppose $(4.11)$ holds in a cone $\Gamma^{\prime} \subseteq R^{m-1}$ containing $\xi_{0}^{\prime}$. 
Let $z_{0}=\left(z_{0}^{\prime}, z_{0}^{m}\right) \in C^{m-1} \times C$ and $\xi=\left(\xi^{\prime}, \xi_{m}\right) \in R_{m-1} \times R$. In order to examine $\mathrm{WF}_{\text {ha }} u$ at $\left(0,\left(\xi_{0}^{\prime}, \xi_{m}\right)\right)$, we have to estimate the FBI:

$$
\begin{aligned}
F\left(z_{0}, \xi\right)=\int_{|t| \leq t_{0}} \int_{\left|x^{\prime}\right| \leq T} \exp \left(\sqrt{-1}\left\langle z_{0}-Z\left(x^{\prime}, t\right), \xi\right\rangle\right. & \left.-|\xi|\left(z_{0}-Z\left(x^{\prime}, t\right)\right)^{2}\right) u\left(x^{\prime}, t\right) d Z .
\end{aligned}
$$

But since $Z_{m}$ depends only on $t$, we get

$$
\begin{aligned}
F\left(z_{0}, \xi\right)=\int_{|t| \leq t_{0}} \exp \left(\left(z_{0}^{m}-Z_{m}(t)\right) \xi_{m}-|\xi|(\right. & \left.\left.z_{0}^{m}-Z_{m}\right)^{2}\right) \\
& \times I\left(t,|\xi| /\left|\xi^{\prime}\right|, z_{0}^{\prime}, \xi^{\prime}\right) d Z_{m}(t) .
\end{aligned}
$$

We now select $C_{0}$ as follows. Since $\left(0, \xi^{0}\right) \notin$ Char $P$, by Lemma $3.1 \exists$ a constant $C_{0}>1$ such that if $|\xi| \geq C_{0}\left|\xi^{\prime}\right|$, then $F\left(z_{0}, \xi\right)$ decays exponentially for $z_{0}$ near 0 in $C^{m}$.

Let $\Gamma=\Gamma^{\prime} \times R$. Pick $\xi=\left(\xi^{\prime}, \xi_{m}\right) \in \Gamma$. To finish the proof, we consider two cases:

Case (i). $|\xi| \geq C_{0}\left|\xi^{\prime}\right|$. This was just taken care of.

Case (ii). $|\xi| \leq C_{0}\left|\xi^{\prime}\right|$. Then $|\xi|=\left(|\xi| /\left|\xi^{\prime}\right|\right)\left|\xi^{\prime}\right|=\tau\left|\xi^{\prime}\right|$ with $1<\tau<C_{0}$.

Hence (4.11) and (4.12) guarantee the exponential decay of $F\left(z_{0}, \xi\right)$ for $z_{0}$ near 0 in $C^{m}$.

\section{REFERENCES}

1. M. S. Baouendi, C. H. Chang, and F. Treves, Microlocal hypo-analyticity and extensions of CR functions, J. Differential Geom. 18 (1983), 331-391.

2. S. Berhanu, Propagation of hypo-analyticity along bicharacteristics, Pacific J. Math. 138 (1989), 221-232.

3. P. Schapira, Propagation at the boundary and reflection of analytic singularities of solutions of linear partial differential equations, Publ. Res. Inst. Math. Sci. Suppl. 12 (1977), 441-453.

4. J. Sjöstrand, Propagation of analytic singularities for second order Dirichlet problems. III, Comm. Partial Differential Equations 6(5) (1981), 499-567.

5. __ The FBI transform for CR submanifolds of $C^{N}$, Preprint.

6. F. Treves, Ovcyannikov Theorem and hyper-differential operators, Notas de Mat., no. 46, Rio de Janeiro, 1968.

7. ___ Basic linear partial differential equations, Academic Press, New York, 1975. 8. $\frac{1}{1980 .}$. Introduction to pseudodifferential and Fourier integral operators. I, Plenum Press,

Department of Mathematics, Temple University, Philadelphia, Pennsylvania 19122 